\title{
CONSIDÉRATIONS PHYSIQUES SUR L'INFLUENCE DES CORPS EN SUSPENSION DANS L'EAU DANS LES TURBO-MACHINES HYDRAULIQUES
}

\author{
COMMUNICATION FAITE' A LA REUNION DE LA SOCIETE HYDROTECHNIQUE DE FRANCE \\ DU 24 NOVEMBRE 1947
}

\author{
par M. Paul BERGERON \\ Ingénieur des Arts et Manufactures, Répétiteur de l'Ecole Centrale des Arts et Manufactures
}

S'il est difficile de calculer mathématiquement l'influence sur l'écoulemenẗ, dans les turbomachines, de corps en suspension dans l'eau, il est par contre possible d'expliquer physiquement un certain nombre de phénomènes qui, à première vue, peuvent paraître paradoxaux.

Parmi ces phénomènes, le plus curieux est celui de la fatigue des turbines et des pompes. Bien connu des turbiniers qui le constatent au moment de la chute des feuilles, il prend pour les pompiers des proportions considérables dans les problèmes de pompage d'eau d'égout.

Rappelons le phénomène: pour une pompe fonctionnant à vitesse constante, sous hauteurs constantes, le débit diminue dans le temps, ainsi que bien souvent la puissance. Le débit diminuant toujours plus vite que la puissance, le rendement décroît. Le phénomène est plus ou moins rapide; le rendement peut tomber de 10 points en une demi-heure ou une heure, comme il peut s'écrouler de 15 points en quelques minutes. Un simple arrêt de la pompe et en général un simple changement de régime, comme par exemple, fermer la vanne et la rouvrir, sans arrêter la pompe, permet de retrouver les caractéristiques initiales.

Or, il ne peut s'agir d'obturation des roues par cie gros corps, puisque normalement, les machines sont protégées par des grilles ne laissant passer que les corps plus petits que les orifices.

La première hypothèse faite, et encore la plus courante, est que ces corps, bien que plus petits que les orifices, s'accrochent à l'extrémité des aubes et s'y accumulent peu à peu, compromettant ainsi l'écoulement normal. L'explication a pu donner satisfaction dans certains cas. Par exemple, à la première usine d'eau d'égoût de Clichy (Préfecture de la Seine) il se formait à l'œillard des roues un amas compact, appelé "bouchon », constitué de chiffons, papiers, brindilles, feuilles, filaments solidement enchevêtrés. A l'arrêt, un retour d'eau en arrière, ramenait le bouchon dans l'aqueduc d'aspiration oì il était harponné par le personnel. Mais à Clichy, les grilles étaient largement espacées.

Four éviter le phénomène, il fut prévu à l'usine d'Auteuil des grilles à barreaux resserrés qui arrêtaient même la majeure partie des feuilles et des papiers pour ne plus laisser passer que des particules de dimensions en apparence insignifiantes, et en particulier la multitude de perits filaments qui existent toujours dans les eaux d'égoûts.

Le phénomène de fatigue était cependant très important. La formation d'un bouchon même réduit n'étant pas exclue, toutes les précautions étaient prises pour essayer d'en révéler l'exisrence. Mais à chaque arrêt, aussi lent soit-il, il ne restait rigoureusement aucun corps dans la pompe.

Comme il se dégage une grande quantité de gaz dus à la fermentation, l'hypothèse fut faite ci'une poche de gaz de volume croissant accrochée à la pointe de roue dans l'axe de rotation. Un dispositif fut prévu, pour éventuellement aspirer cette poche, qui n'apporta aucune amélioration.

A noter que des couteaux fixes épousant les f́rmes méridiennes de l'arête des aubes à l'entrée (croquis $n^{0}$ 1) ne suppriment pas la 


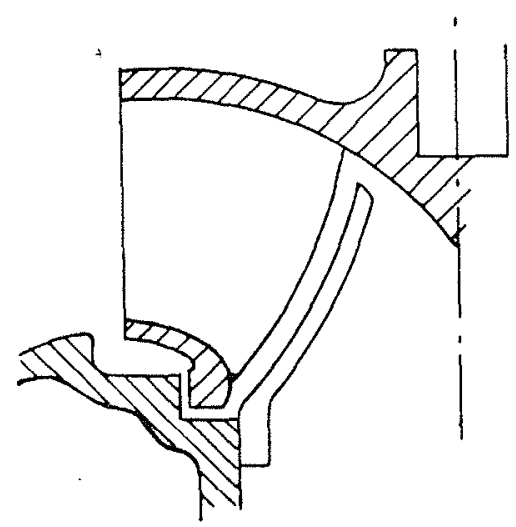

fig. 1

fatigue, ce qui infirme également la première hypothèse.

Or, le pompage de mixture, de sable par exemple, même avec de très fortes concentrations ne permet de relever aucun phénomène analogue à la fatigue. Si la caractéristique des pompes est affectée par la présence de sable dans l'eau, par contre le fonctionnement reste identique à lui-même dans le temps.

Ces différentes considérations permettent de conclure: $1^{\circ}$ que le phénomène de fatigue est dû à la présence de corps plus légers que l'eau ;

$2^{\circ}$ que les corps plus légers peuvent s'accumuler dans la roue tandis que les corps plus lourds sont obligatoirement rejetés.

Il est possible d'en donner une explication physique.

Considérons le cas d'une pompe centrifuge et, pour la facilité de l'exposé, supposons l'écoulement entre deux faces parallèles. En un point $A$ quelconque (fig. 2) l'élément fluide ou solide qui s'y trouve est soumis aux accélérations dW $W^{2} \quad U^{2}$

- $\frac{\text { dt }}{2 \omega} \mathrm{W},-$ et $\frac{}{\mathrm{R}}$ qui sont équilibrées dans toutes les directions par la différence de pression dans cette direction.

$W$ étant la vitesse relative, $U$ la vitesse d'entrainement, (1) la vitesse angulaire de rotation, $\rho$ le rayon de courbure de la trajectoire, $R$ le rayon du point considéré par rapport à l'axe de rotation

La différence de pression est nulle dans la direction pour laquelle la somme des projections

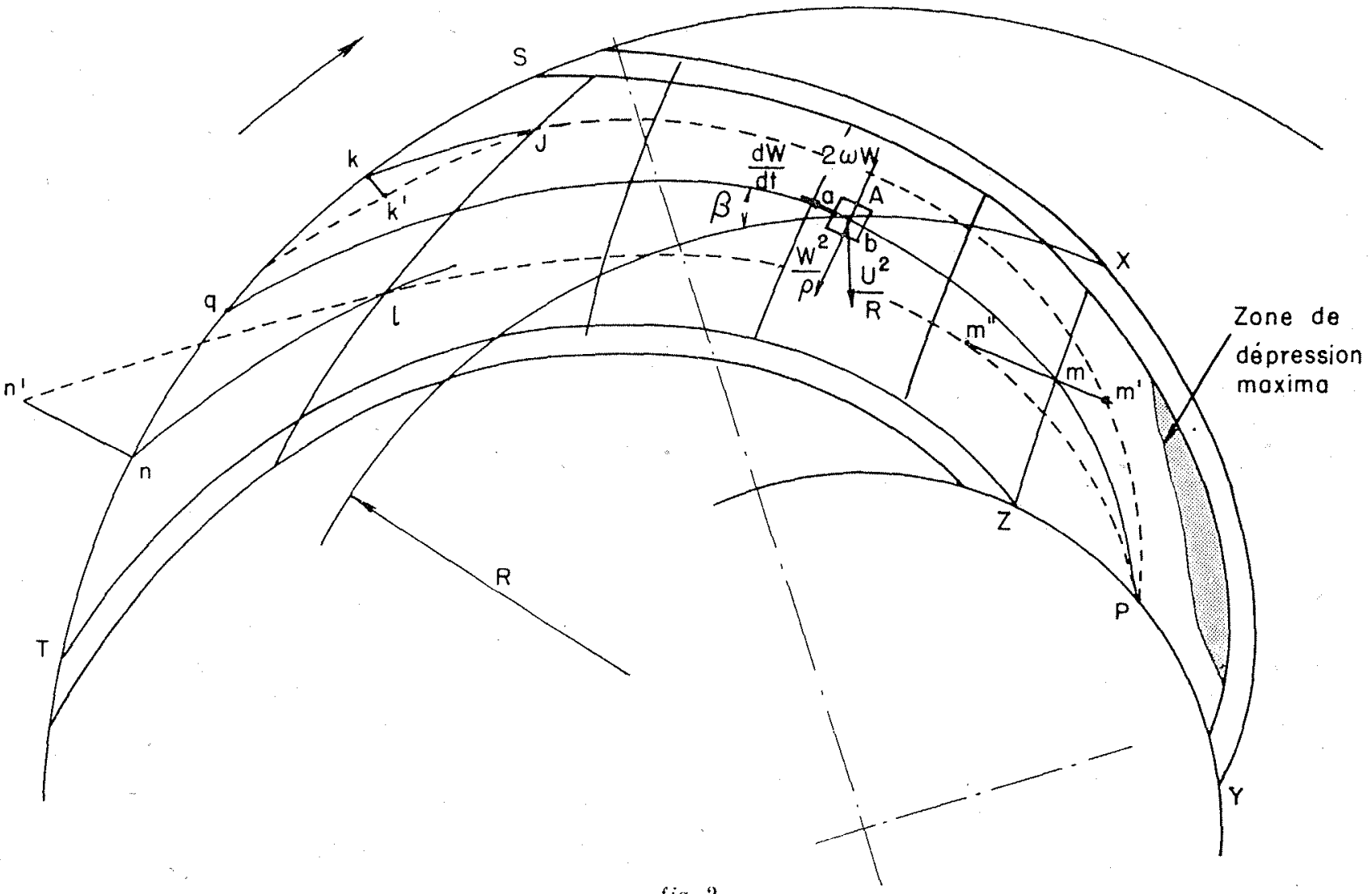

fig. 2 
de toutes les accélérations est nulle. Le maximum de différence de pression est pour la direction perpendiculaire à cette dernière. Supposons en A un petit cube orienté suivant ces directions. Si ce cube est de l'eau, il existera entre les faces $a$ et $b$ une différence de pression maximum résultant de l'équilibre entre les différentes accélérations et cette différence de pression. Dans le cas où ce cube est un corps plus léger que l'eau, et en supposant qu'il soit entraîné à la même vitesse et dans la même direction que l'eau qui l'entoure, comme il est soumis aux mêmes accélérations, il ne peut compenser qu'une différence de pression qui, par rapport à celle de l'eau est proportionnelle à sa densité. Dans ce cas, le corps plus léger est donc soumis à un effort de glissement par rapport à l'eau, dirigé vers la zone de plus faible pression, dans une direction perpendiculaire à la ligne d'équipression, proportionnel à la différence de densité "et à l'accélération résultant du milieu. Le champ d'accélération étant considérablement plus grand que celui de la pesanteur, il en résulte que le corps tend à glisser par rapport à l'eau avec un effort beaucoup plus grand que celui qui, dans le champ terrestre, sollicite un corṕs immergé à monter vers la surface.

Par contre, il est difficile de calculer exactement cet effort, car dès que le corps glisse par rapport à l'eau, les vitesses $W$ du corps, et de l'eau qui l'environne, ne sont plus les mêmes et les accélérations sont également différentes.

Par contre, il est possible de déterminer l'ordre de grandeur du phénomène en établissant les équations d'équilibre du corps suivant la tangente à la trajectoire liquide et suivant la normale à cette trajectoire, sous l'effet des accélérations, des différences de pression et de la résistance dûe au glissement du corps par rapport à l'eau, eir tenant compte que si la trajectoire liquide est connue, les pressions auxquelles est soumis le corps sont également connues.

Reprenons le raisonnement pour un corps plus lourd que l'eau. L'effort qu'il appliquerait à l'eau s'il suivait rigoureusement la trajectoire serait supérieur à la différence de pression dûe à l'eau; il glissera donc par rapport à l'eau, avec un effort dirigé vers la zone de plus forte pression, et comme pour le corps plus léger, dans une direction perpendiculaire à la ligne d'équipression, l'effort étant proportionnel à la différence de densité et à l'accélération résultant de l'écoulement.
Grâce à cette observation, il est possible d'avoir une idée des trajectoires suivies par des corps plus légers ou plus lourds que l'eau, par rapport à celles suivies par l'eau.

D'une manière générale, il existe, dans les pompes, une zone de dépression par rapport à la pression à l'œillard, localisée à l'arrière des aubes et vers l'entrée, zone indiquée $X Y$ sur la figure 2. A l'avant des aubes et à l'entrée, en $Z$, la pression est au moins égale à celle dans l'œillard. La ligne équipression a donc l'allure générale $Z X$.

A la sortie de la roue, les pressions sont théoriquement égales sur tout le pourtour. La ligne équipression à la sortie est donc sensiblement donnée par la circonférence ST. Entre ces deux extrêmes, les lignes équipressions varient progressivement.

Soit une trajectoire liquide $p$ q. Si un corps part de $p$ en même temps qu'une molécule liquide, lorsque cette dernière sera en $m$, si le corps est plus léger que l'eau, il aura glissé normalement aux surfaces équipressions et sera en $\mathrm{m}^{\prime}$; s'il est plus lourd, il sera en $\mathrm{m}^{\prime \prime}$.

La trajectoire des corps plus légers coupe donc les lignes de courant et s'incurve à l'entrée vers les zones de faibles pressions c'est -à-dire vers l'arrière de l'aube, celle des corps plus lourds coupe également les lignes de courant, mais s'incurve à l'entrée vers l'avant des aubes, c'està-dire vers les zones de fortes pressions.

Si les corps n'ont pas rencontré les parois, le plus léger se retrouvera en i, le plus lourd en 1 .

En j la trajectoire liquide étant $j k$, le corps plus léger décrira $j k^{\prime}$, ayant glissé de $k k^{\prime}$ par rapport au liquide. II sortira donc avec une trajectoire plus couchée que celle de l'eau. Pour le corps plus lourd, sa trajectoire sera $n^{\prime}$ si celle de l'eau est $n$, c'est-à-dire qu'elle sera plus redressée que celle de l'eau.

Voyons ce qui se passe lorsque la trajectoire d'un corps rencontre la paroi, et tout d'abord dans le cas d'un corps plus léger que l'eau. Tant que le corps est en mouvement, il est sollicité à se diriger vers la paroi. S'il colle à la paroi le corps est brusquement entraîné à la vitesse de rotation $U$ et n'est plus scumis qu'à une seule $U^{\circ}$ accélération - qui se décompose en une accé- 


$$
\begin{aligned}
& \text { lération normale à la paroi } \frac{U^{2}}{R} \cos \beta \text { et à une } \\
& \text { accélération tangentielle } \frac{U^{2}}{\mathrm{R}} \sin \beta \text { (fig. 3). }
\end{aligned}
$$$$
R
$$

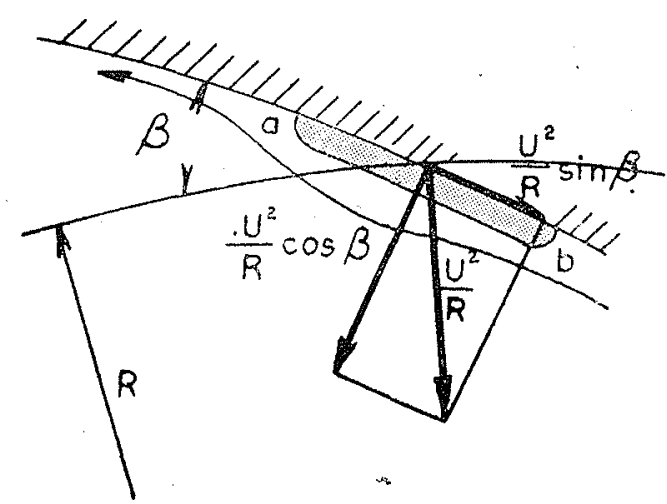

fig. 3

En outre, il est soumis à une différence dé pression entre $a$ et $b$ et à un effort de frottement de l'eau contre sa face extérieure (fig. 3).

L'accélération normale $\frac{U^{2}}{R} \cos \beta$ est communiquée par la paroi contre laquelle le corps 'applique. L'accélération $\frac{U^{2}}{R} \sin \beta$ ne peut être communiquée que par la différence de pression entre a et b et par l'effort de frottement sur la paroi qui doit vaincre également l'effort d'entraînement dû au courant.

Comme il s'agit en général de corps plats et minces (feuilles, papiers, etc...) le coefficient de frottement est très élevé. D'autre part, $\beta$ est souvent inférieur à $30^{\circ}$, si bien que la composante normale est nettement plus grande que la composante horizontale. Bien que la différence de pression entre $a$ et $b$ n'intervienne pas pour ces corps plats, la seule condition d'un frottement élevé peut expliquer que le corps reste collé à la paroi et ne puisse plus s'en détacher.

Une première couche est ainsi constituée, formant elle-même une surface plus rugueuse, sur laquelle viennent se déposer des corps qui nourrissent le dépôt. La rugosité superficielle crcissant, des corps plus importants peuvent être arrêtés, comme par exemple des bouchons, qui au début auraient échappé. Le dépôt augmentant, à l'effort de frottement qui fournissait l'accélé- ration $\frac{U^{2}}{R} \sin \xi$, vient s'ajouter la différence de

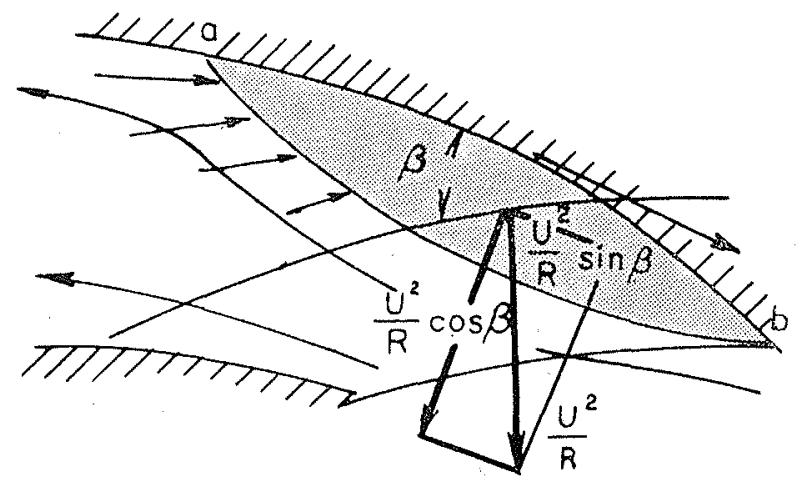

fig. 4

pression dûe à l'écoulement (fig. 4), ce qui autorise des dépôts de plus en plus importants jusqu'à un volume et une forme limite pour lesquels aucun corps ne se dépose plus, par suite de la modification des courbes équipressions, et $U$ pour lesquels l'équilibre existe entre $\frac{-}{R} \sin \hat{\beta}$ : les forces de frottement et les différences de pression entre $a$ et $b$.

L'importance de ce dépôt justifie une modification de l'écoulement et par conséquent, une modification des caractéristiques, ainsi qu'un lâcher de tourbillons provoquant la chute du rendement.

Pour une variation importante du régime, par exemple un fonctionnement vanne fermée, la répartition des vitesses et surtout des pressions change totalement. Le dépôt qui constituait un b'oz compact sous l'effet des accélérations et des pressions cesse alors d'être en équilibre et se détache de la paroi en s'effritant. En cas d'arrêt di: groupe, la disparition de l'accélération $\frac{U}{R}$ justifie facilement ce détachement.

Examinons maintenant le cas où la trajectoire d'un corps plus lourd que l'eau rencontre la paroi. Ce sera obligatoirement la face avant de l'aube. Si le corps rencontrant la paroi s'arrêtait,

if ne serait plus soumis qu'à l'accélération $\frac{U}{R}$,

toutes les autres s'annulant, accélération qui se décompose comme précédemment en une accé$U^{2}$

lération normale - $\cos \beta$ et une tangentielle $\mathrm{R}$. 
$\bigcup^{2}$

- $\sin \beta$. Or il est visible que l'accélération

$R$

$U^{\prime \prime}$

$-\cos \xi$ ne peut lui être fournie par aucune

différence de pression et que, même si le corps se trouvait arrêté véritablement par un défaut de fonderie, il s'échapperait de la paroi sous l'effet de sa force d'inertie. Pour retrouver un équilibre, il est nécessaire que les autres accélérations ne s'annulent pas, et en particulier 2 (s) $W$, qui U:

peut seule équilibrer $-\cos \beta$; le corps conti-

nue donc à avancer, en glissant sur la paroi avec une certaine vitesse $W$ plus faible que celle qu'avait le corps avant de toucher la paroi et telle que accélération, différence de pression, force de frottement sur la parói et résistance dûe au glissement du corps par rapport à l'eau, s'équilibrent.

Lorsque le corps glisse sur la paroi, il est également possible 'd'avoir une idée de l'ordre de grandeur de l'effort appliquant ce corps contre la paroi, car la trajectoire de l'eau et celle du corps sont les mêmes. On connaît donc parfaitement les angles et les rayons de courbure. Si la vitesse de l'eau est connue, et par conséquent les différences de pression qui en résultent autour du corps, en écrivant l'équilibre des accélérations et des forces sur la tangente, puis sur la normale à la trajectoire, on obtient deux équations dont les inconnues sont la vitesse du corps et l'effort normal à la paroi.

Les résultats ainsi obtenus ne peuvent être que très approximatifs. Ils permettent toutefois dobserver que les efforts des corps contre la paroi peuvent être réduits. On peut conclure également très grossièrement que les corps cessent de toucher la paroi lorsque les courbes équipressions font avec la paroi prise dans le sens de l'écoulement, un angle supérieur à $90^{\circ}$, et qu'ils sont éjectés sans toucher à l'extrémité des aubes.

A l'appui de ces remarques, on peut signaler lo cas d'une roue de pompe centrifuge qui, après le pompage de 1.000 .000 de $\mathrm{m}^{3}$ de sable présentait les usures suivantes:

- arêtes d'entrée des aubes reculées par usure de plusieurs centimètres.

- - faces avant des aubes usées très régulièrement de plusieurs millimètres.
- arêtes de sortie affutées à l'origine, restées rigoureusement affutées et au même diamètre.

Le fait que les corps sortiraient de la roue sans toucher aux aubes pourrait expliquer également le phénomène curieux constaté sur les pompes ¿̀ déblais où il n'y a pratiquement jamais coïncement de pierres entre le rouet et la languette du colimaçon, même lorsque l'espace laissé libre est beaucoup plus faible que la dimension des matériaux pompés.

Tous les raisonnements qui précèdent ont été faits en considérant l'écoulement dans les pomues. Des raisonnements analogues pourraient être faits pour l'écoulement dans les turbines.

Les accélérations 2 $\omega, \frac{W^{2}}{\rho}, \frac{V^{2}}{R}$ se pré-

seritent comme pour la pompe, seul $\frac{d W}{d t}$ est

inversé. On trouve également que les corps plus légers sont déviés à l'entrée vers les zones de faibles pressions où ils peuvent s'accumuler lorsqu'ils sont collés à la paroi. De même, les corps plus lourds sont déviés à l'entrée vers les zones de fortes pressions et ne peuvent rester sur la paroi. La différence importante réside dans le fait que dans les pompes, les corps plus lourds ont une vitesse supérieure à celle de l'eau, tandis que pour les turbines la vitesse de ces corps est inférieure; et inversement pour les corps plus légers. Dans les pompes, la concentration en corps plus légers est donc supérieure à la concentration moyenne, et pour les corps plus lourds, inférieure à celle-ci. Le phénomène est inverse dans les turbines.

En ceci, la pompe peut être comparée à un divergent dans lequel les bulles d'air ont tendance à s'accumuler à la petite section, tandis que les graviers le traversent plus rapidement que l'eau; la turbine peut être comparée au convergent dans lequel les bulles d'air vont plus vite que l'eau et où les graviers se concentrent.

Enfin d'une manière très générale, vérifiée en appliquant dans chaque cas les formules d'équilibre des corps plus lourds ou plus légers que l'eau dans le milieu où ils se trouvent, on peut dire en simplifiant tout raisonnement que les corps les plus lourds glissent vers les zones de plus fortes pressions, et les corps plus légers vers les zones de plus faibles pressions en s'y accumulant, ou en s'en échappant suivant les cas, et ceci plus ou moins aisément.

Un prochain numéro donnera l'échange de vues qui suivit cet exposé. 


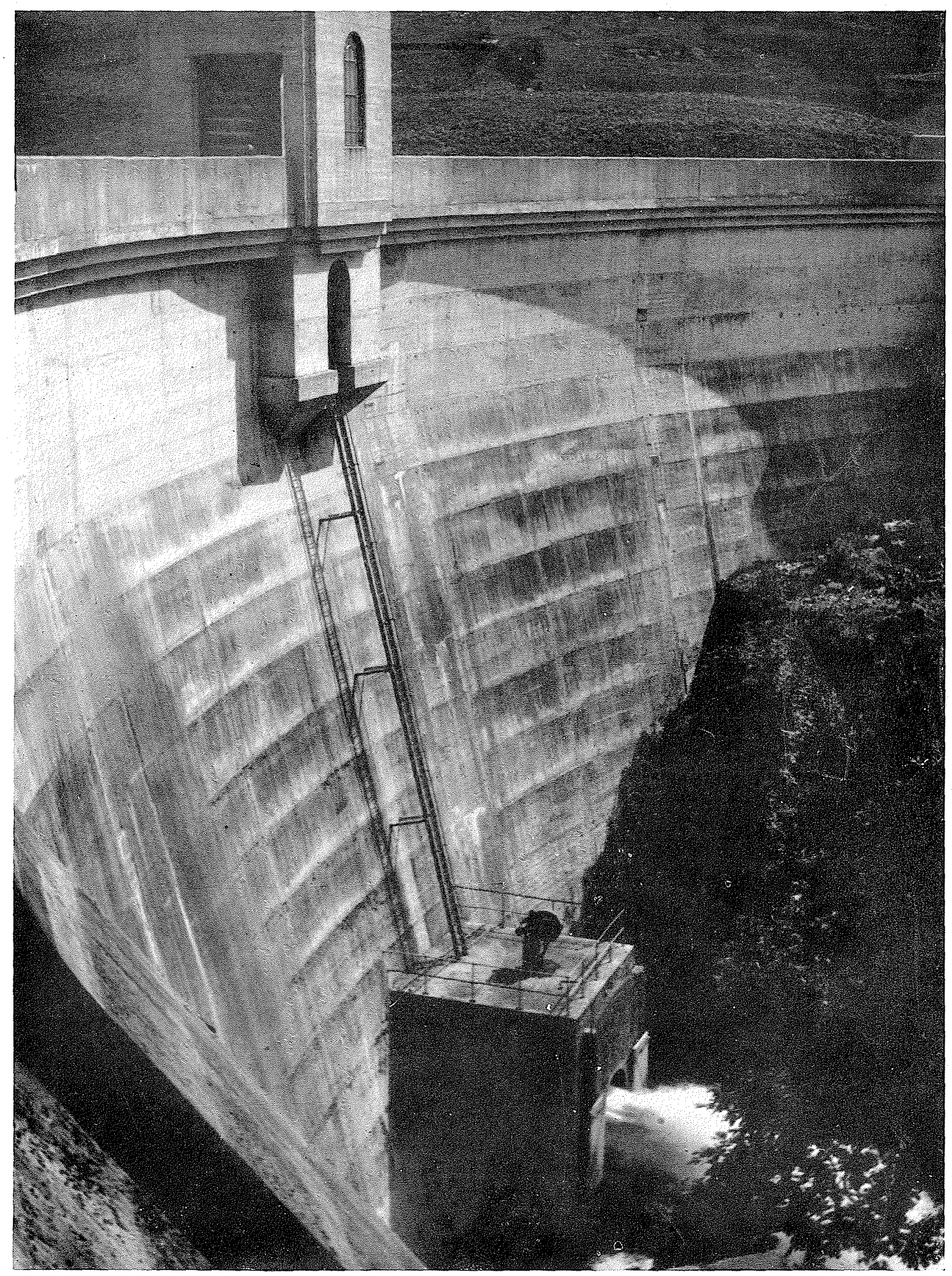

VUE DÉTAILLEE DU BARRAGE KEN, DANS LE SYSTEMmE CALLOWAY (Ecosse)

(Photo British Council) 